\title{
CHLORAL HYDRATE: A POTENTIAL CARCINOGEN
}

The long-term health effects of chloral hydrate are discussed and its carcinogenicity in mice are reported from the University of California, Davis, and the California School of Public Health, Berkeley. In a group of 8 mice receiving $10 \mathrm{mg} / \mathrm{kg}$, 6 developed hepatic adenomas or carcinomas, an incidence significantly greater than that of $2 / 19$ controls with carcinomas. Of 24 mice receiving $166 \mathrm{mg} / \mathrm{kg} /$ daily intake of chloral hydrate in drinking water for 104 weeks, 17 developed hepatic adenomas or carcinomas compared to 3 of 20 controls. Other recent studies have shown chloral hydrate to be genotoxic, causing chromosome changes in vivo and in vitro. (Salmon $A G$, Kizer KW et al. Potential carcinogenicity of chloral hydrate - a review. Clin Toxicol 1995;33:115-121). (Respond: Dr Kenneth Kizer, Department of Community and International Health, School of Medicine, TB-168, University of California, Davis, CA 95616).

COMMENT. Many of us who have stocked chloral hydrate in large quantities for use in sedating children for EEGs and other short procedures may want to reconsider its safety and substitute an alternative. Certainly, its chronic long-term use as a sedative in mentally retarded children should be discouraged until further studies are completed. Chloral hydrate is a metabolite of trichloroethylene, a known carcinogen.

\section{HEREDO-METABOLIC DISORDERS}

\section{PRESENTING SIGNS OF MITOCHONDRIAL DISEASE}

Clinical features of 51 patients confirmed with mitochondrial respiratory chain disease and clinical investigations most helpful in diagnosis of different phenotypes are reported from the Divisions of Clinical Neuroscience and Neurobiology, University of Newcastle upon Tyne, UK. Ages ranged from birth to 55 years, and 21 patients were $<16$ years. Presenting symptoms in order of frequency included ptosis and ophthalmoplegia (20), lactic acidosis (10), seizures (6), myopathy (6), failure to thrive (6), and ataxia (5). Features other than ptosis and ophthalmoplegia identified as clues to respiratory chain dysfunction were as follows: 1) lactic acidosis with deafness, short stature/failure to thrive, or basal ganglia calcifications on CT; 2) family history of neurological disease with maternal transmission; and 3) proximal myopathy and CNS disease. In addition to well-recognized syndromes (MERRF and MELAS) many had non-specific encephalopathies. The most useful confirmatory diagnostic test was histochemical analysis of muscle. (Jackson MJ, Bindoff LA et al. Presentation and clinical investigation of mitochondrial respiratory chain disease. A study of 51 patients. Brain April 1995;118:339-357). (Respond: Dr LA Bindoff, Division of Clinical Neuroscience, The Medical School, University of Newcastle upon Tyne, Framlington Place, Newcastle upon Tyne NE2 4HH, UK).

COMMENT. Mitochondrial respiratory chain disease is manifested by a large variety of syndromes, but histological and chemical analysis of skeletal muscle is frequently diagnostic. Succinate dehyrogenase, cytochrome c oxidase activity, and DNA studies in muscle may be performed on a needle biopsy specimen. Elevated CSF lactate is a good 
indicator of mitochondrial disease in patients with encephalopathic disorders.

The treatment of congenital lactic acidosis is reviewed from the Center for Inherited Disorders of Energy Metabolism, Case Western Reserve University, Cleveland, OH (Kerr DS. Int Pediatr 1995;10:75-81). Treatments have included diet, vitamins, use of enzyme activators, and enzyme replacement. None has been very successful.

See Progress in Pediatric Neurology I and II (Millichap JG, Ed.

PNB Publ, 1991 and 1994) for further articles on mitochondrial cytopathies. The diagnosis of mitochondrial disorder should be considered with the following: 1) an unexplained association of symptoms; 2) an early onset and rapidly progressive course; and 3) involvement of unrelated organs sharing no common embryologic origin and no common biological functions.

\section{PRENATAL DIAGNOSIS OF LESCH-NYHAN SYNDROME}

The results of carrier and prenatal diagnosis for Lesch-Nyhan syndrome by carrier testing of 83 women and prenatal analysis of 26 pregnancies are reported from the Department of Molecular and Human Genetics, Baylor College of Medicine, Houston, TX. Mutation detection and linkage analysis were used for probands and their families and biochemical measurement of HPRT enzyme activity for at-risk pregnancies. Mutations in the HPRT gene of affected males were detected in $100 \%$ cases. Forty five $(56 \%)$ at-risk women were found not to carry their family's HPRT gene mutation. (Alford RL et al. Lesch-Nyhan syndrome: Carrier and prenatal diagnosis. Prenat Diagn April 1995;15:329-338). (Respond: Dr RL Alford, Department of Molecular and Human Genetics T-528, Baylor College of Medicine, One Baylor Plaza, Houston, TX 77030).

COMMENT. Lesch-Nyhan syndrome is an X-linked recessive disorder characterized by hyperuricemia, choreoathetosis, mental retardation, self-mutilatory behavior, and a deficiency of the enzyme hypoxanthine guanine phosphoribosyltransferase (HPRT). Molecular diagnostic studies of affected males and carrier testing prior to pregnancy have been shown to demonstrate genetic risks and unnecessary prenatal tests may be avoided

\section{5-HYDROXYTRYPTOPHAN IN FRIEDREICH'S ATAXIA}

The effect of the levorotatory form of 5-hydroxytryptophan (approx 1 $\mathrm{gm} /$ day/orally) on cerebellar symptoms in 26 patients with Friedreich's ataxia was evaluated in a double-blind drug-placebo study by the Ataxia Research Center, Hopital Neurologique, Lyon and 11 other research hospitals in France. Of 19 completing the study, 11 were treated with 5-hydoxytryptophan and 8 with placebo. A significant decrease of the kinetic score and improvement in coordination was observed in the active treatment group after 6 months but not at 4 months, indicating a progressive drug effect. Five subtests demonstrating improvement included finger-nose test, heel-knee, and Archimedes' spiral. A trend toward acceleration of the speed of speech was also observed. Gastrointestinal symptoms were the main side-effects of treatment. (Trouillas P et al. Levorotatory form of 5-hydroxytryptophan in Friedreich's ataxia. Arch Neurol May 1995;52:456-460). (Reprints: Dr Trouillas, Ataxia Research Center and Cerebrovascular Unit, Faculte Alexis Carrel, Universite Claude Bernard, Hopital Neurologique, 59 Boulevard Pinnel, 69003 Lyons, France). 